\title{
Bunje (Novo Selo, Croatie)
}

Campagne 2018

Emmanuel Botte, Audrey Bertrand, Kristina Jelinčić, Bastien Lemaire, Nicolas Leys, Antoine Boisson et Alexia Rozak

\section{(2) OpenEdition \\ 12 Journals}

Édition électronique

URL : http://journals.openedition.org/cefr/3481

DOI : $10.4000 /$ cefr.3481

ISSN : 2282-5703

Éditeur

École française de Rome

Référence électronique

Emmanuel Botte, Audrey Bertrand, Kristina Jelinčić, Bastien Lemaire, Nicolas Leys, Antoine Boisson et Alexia Rozak, "Bunje (Novo Selo, Croatie) », Chronique des activités archéologiques de l'École française de Rome [En ligne], Balkans, mis en ligne le 05 septembre 2019, consulté le 07 septembre 2019. URL : http://journals.openedition.org/cefr/3481; DOI : 10.4000/cefr.3481

Ce document a été généré automatiquement le 7 septembre 2019.

(c) École française de Rome 


\title{
Bunje (Novo Selo, Croatie)
}

\author{
Campagne 2018
}

Emmanuel Botte, Audrey Bertrand, Kristina Jelinčić, Bastien Lemaire, Nicolas Leys, Antoine Boisson et Alexia Rozak

\section{Introduction}

1 Le chantier de Bunje sur l'île de Brač constitue la première étape d'un programme portant sur les villae de l'Adriatique orientale, conduit par E. Botte, A. Bertrand et K. Jelinčić et intitulé "Recherches sur l'exploitation économique de la Dalmatie à l'époque romaine (II ${ }^{\mathrm{e}}$ s. av. J.-C. - $\mathrm{II}^{\mathrm{e}}$ s. apr. J.-C.) ». L'intention des auteurs de ce projet est de mesurer, à travers des opérations de fouilles et de prospections, l'impact économique de la présence romaine en Dalmatie ${ }^{1}$.

2 La première campagne menée en 2015 sur le site de Bunje (commune de Novo Selo) sur l'île de Brač était destinée à obtenir une vision d'ensemble de l'ampleur du site et de l'état de préservation de ses vestiges (fig. 1). Il en était ressorti un site assez étendu (les vestiges couvrant un espace de près de $15000 \mathrm{~m}^{2}$, et la villa couvrant à l'intérieur de celui-ci près de $2500 \mathrm{~m}^{2}$ ) et dont les structures sont dans l'ensemble assez bien conservées, ce qui confirme les observations effectuées lors d'un premier rapport mené par K. Jelinčić en 2012 (fig. 2)2. Le site est inscrit au registre des monuments culturels sous le numéro 486, et protégé en tant que tel auprès de la Surintendance de Split par décision du 26 septembre 1979 (sous le numéro 17/41-1979). 
Fig. 1 - Carte de situation de lîle de Brač et de la commune de Novo Selo.

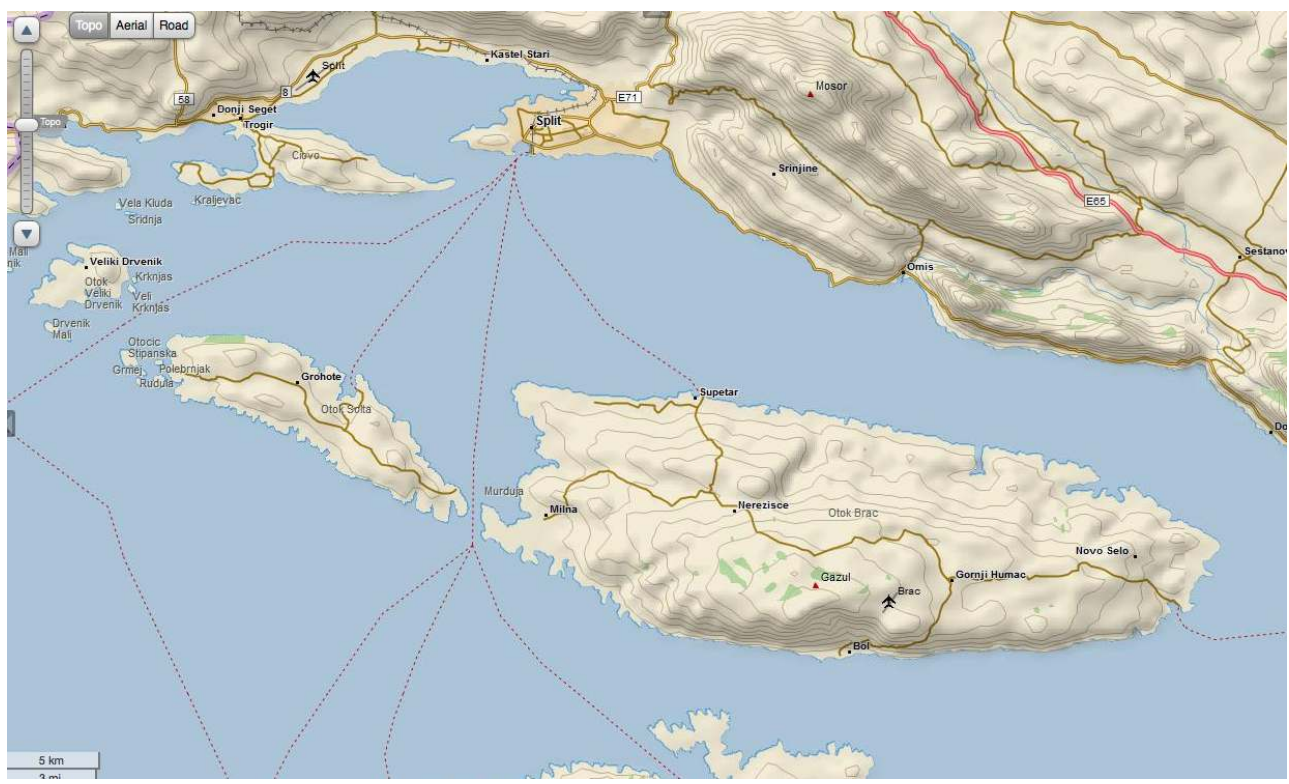

(c) GoogleMaps.

Fig. 2 - Vue du site par drone au début de la campagne.

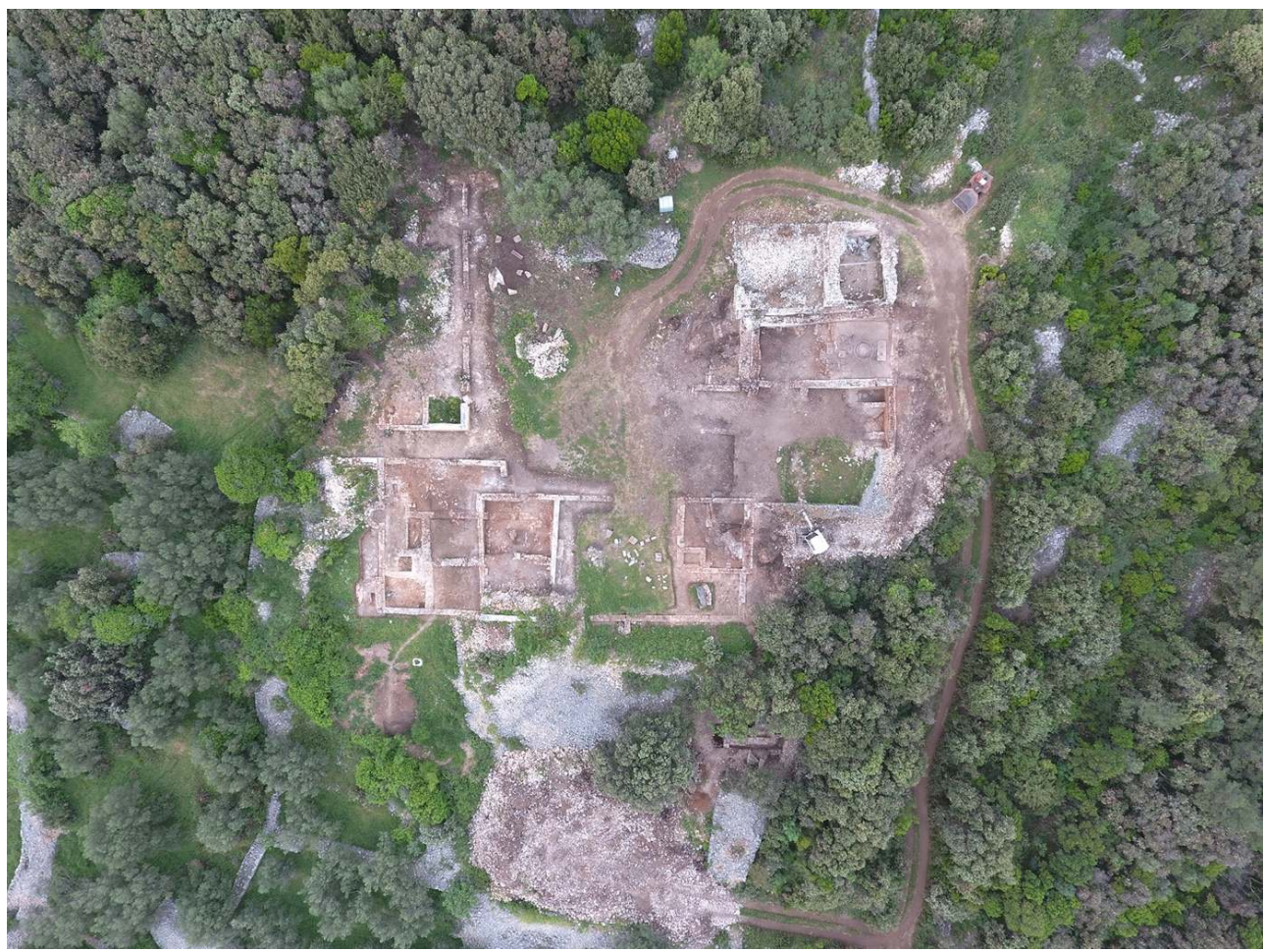

Cl. E. Botte.

3 Les opérations menées sur ce site s'inscrivent dans une recherche plus large visant à clarifier les mécanismes et la chronologie de l'occupation du territoire de la Dalmatie centrale en amont et en aval de la conquête romaine de ce territoire à la fin de la période tardo-républicaine. Au sein de cette problématique, les fouilles de la villa de Bunje nous 
permettront de déterminer le cadre historique et environnemental qui accueille cette structure, ainsi que l'organisation de sa production.

En 2017 et avril 2018, les campagnes de fouilles nous avaient permis de faire des avancées significatives dans la compréhension du plan de la villa, en faisant apparaitre la partie résidentielle (pars urbana) et la partie productive (pars rustica) de part et d'autre d'une grande cour centrale. Nous avions pu notamment compléter le plan dans les secteurs méridionaux et orientaux, faisant apparaître un bâtiment au sud-est des structures connues, ainsi qu'un pressoir à huile dans un très bon état de conservation.

\section{Déroulement et objectifs de la campagne d'octobre 2018}

5 La campagne de fouilles s'est déroulée du 15 au 26 octobre 2018, sous la direction d'E. Botte, A. Bertrand et K. Jelinčić Vučković. L'équipe de fouille était constituée d'A. Boisson, B. Lemaire, N. Leys et A. Rozak.

La campagne a été financée par l'École française de Rome, le Ministère de l'Europe et des Affaires étrangères, le Centre Camille Jullian, le Ministère de la Culture de Croatie et la municipalité de Selca. Les mobiliers en cours d'étude et ceux considérés comme importants pour la datation et l'histoire du site ont été consignés à l'Institut archéologique de Zagreb. L'étude archéozoologique est réalisée par Siniša Radović (Académie des sciences et des arts à Zagreb); l'étude anthracologique par Christophe Vaschalde (Université Paul Valéry-Montpellier 3) ; l'étude paléobotanique par Margaux Tillier (Université Paul Valéry - Montpellier 3) ; l'étude micro-morphologique des sols par Cristiano Nicosia (Université de Padoue); l'étude du verre par Bartul Siljeg (Institut Archéologique de Zagreb); les études anthropologiques par Mario Novak (Institut d'Anthropologie de Zagreb); les études numismatiques par Anja Bertol (Université de Zagreb) ; les analyses chimiques par Nicolas Garnier (Laboratoire Nicolas Garnier). Nicolas Leys, responsable de la photogrammétrie sur le site, a fait une modélisation 3D du site et des principaux blocs de pierre. Les relevés topographiques et les plans ont été réalisés par K. Jelinčić Vučković. L'étude du mobilier céramique a été réalisée par E. Botte et les dessins par A. Boisson.

6 Les objectifs de la campagne d'octobre étaient concentrés sur le secteur du pressoir (secteur 8) et celui du fouloir et de son bassin de récolte (secteurs 6 et 7) (fig. 3). Nous souhaitions en effet comprendre l'organisation des espaces entre le secteur du fouloir et du pressoir, et tenter d'intercepter la forme et l'organisation d'un potentiel chai. 
Fig. 3 - Plan de la villa avec les secteurs fouillés durant cette campagne (situation avant la fouille).

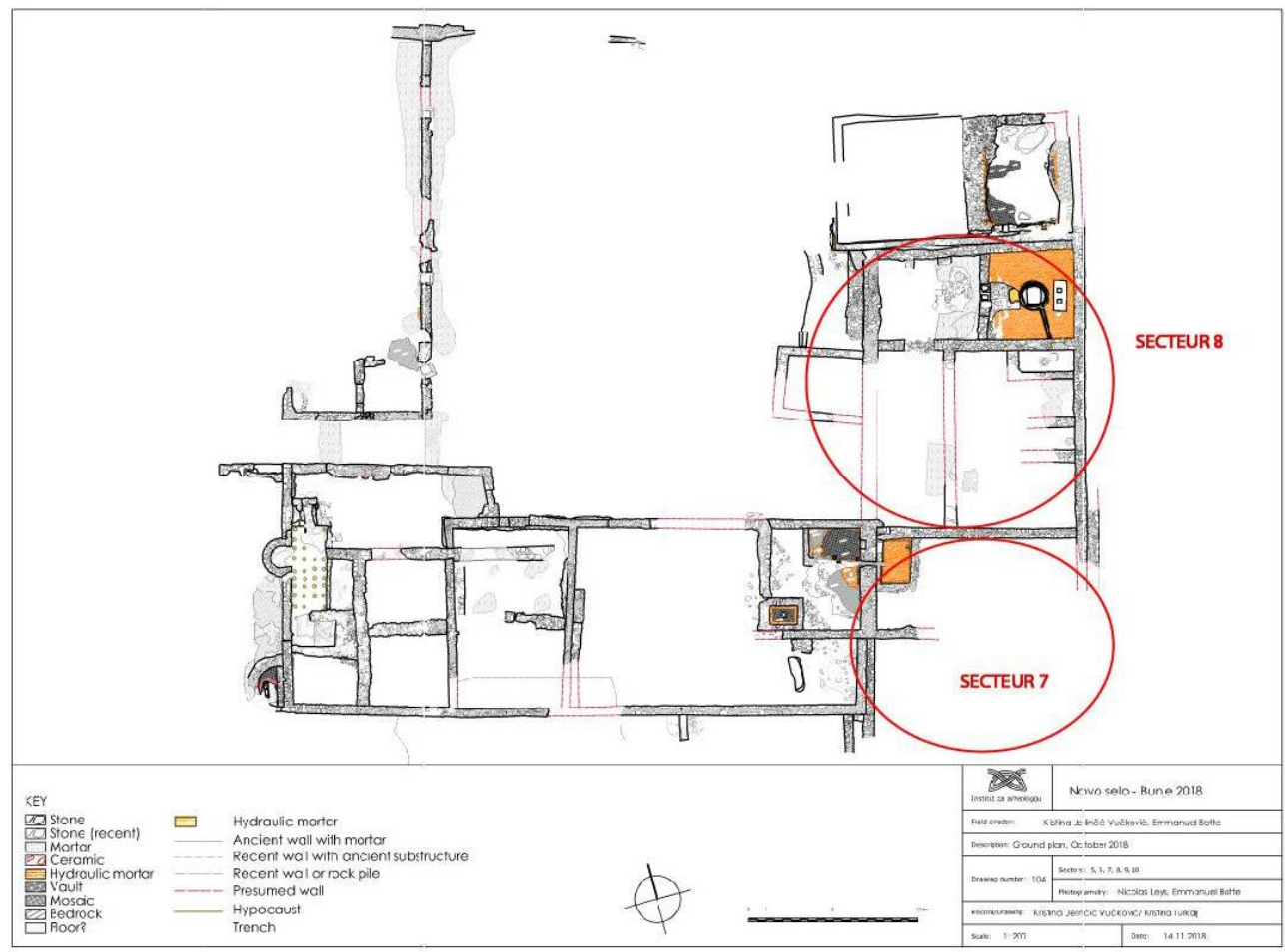

Plan K. Jelinčić.

Une grande partie du travail de cette brève campagne a consisté, comme c'est désormais l'habitude sur ce site, à démanteler les murs modernes à l'aide d'une pelle mécanique mise à disposition par la mairie de Selca. En effet, le site ayant fait l'objet de cultures agricoles depuis près d'un siècle, les différentes générations ont constitué de grands amas de pierres (gomila) pouvant atteindre près de $2 \mathrm{~m}$ de hauteur et qui gênent considérablement la lecture dans son ensemble. Bien entendu une grande partie de ces amas de pierres et du mobilier archéologique qui s'y trouve correspond au démantèlement des structures archéologiques de la villa antique. De ce fait, le démontage de ces structures est réalisé systématiquement en présence de plusieurs membres de l'équipe, le mobilier conservé et les pierres travaillées mises de côté en vue de la restauration du site et de sa présentation au public lorsque les fouilles seront achevées.

\section{Résultats}

\section{Secteur 7}

Une partie de la campagne a été dédiée au dégagement du secteur 7, dont nous savions qu'il abritait au moins le bassin de récolte (BS 7002) du jus de raisin provenant du fouloir situé de l'autre côté du mur MR 6044, une canalisation (CN 6034) reliant ces deux structures. Nous avons ainsi mis au jour un bassin en béton de tuileau $(2,65 \times 1,75 \mathrm{~m})$ qui a livré, comme nous l'avons déjà constaté sur les autres structures de production du site, deux phases de fonctionnement (fig. 4). La plus ancienne est caractérisée par un sol mosaïqué dans un état de conservation très usé, puisque les tesselles ont la forme dite en pointe-de-diamant, ce qui est en général attribué à l'effet de l'acide oléique, ce qui ne 
manque pas de surprendre pour un bassin censé récolter le jus du fouloir (fig. 5). La seconde phase est marquée par un sol en béton de tuileau coulé directement sur le sol précédent, d'une épaisseur d'environ $10 \mathrm{~cm}$ (fig. 6).

Fig. 4 - Vue depuis le nord du bassin de récolte (BS 7002) du jus de raisin provenant du fouloir.

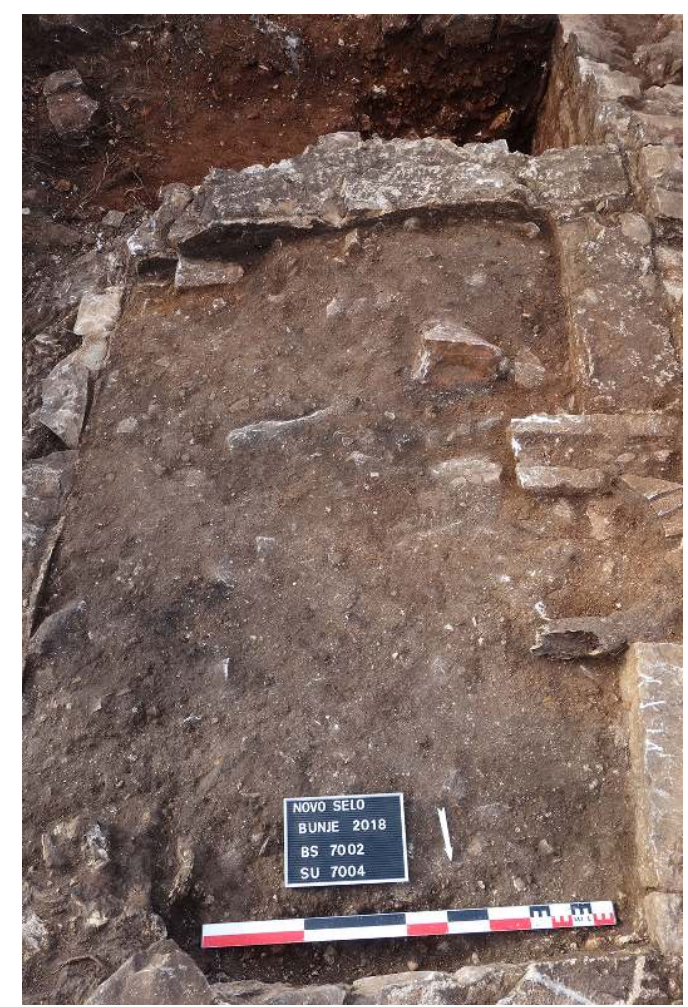

Cl. E. Botte. 
Fig. 5 - Vue du sol en mosaïque du bassin BS 7002.

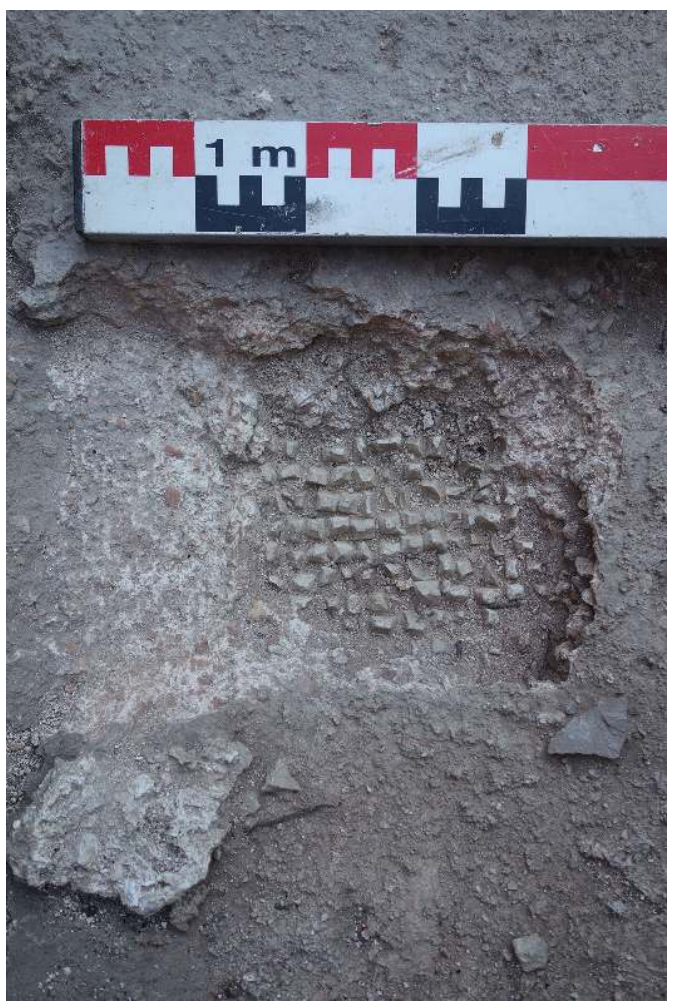

Cl. E. Botte.

Fig. 6 - Vue du sol en béton de tuileau du bassin BS 7002 .

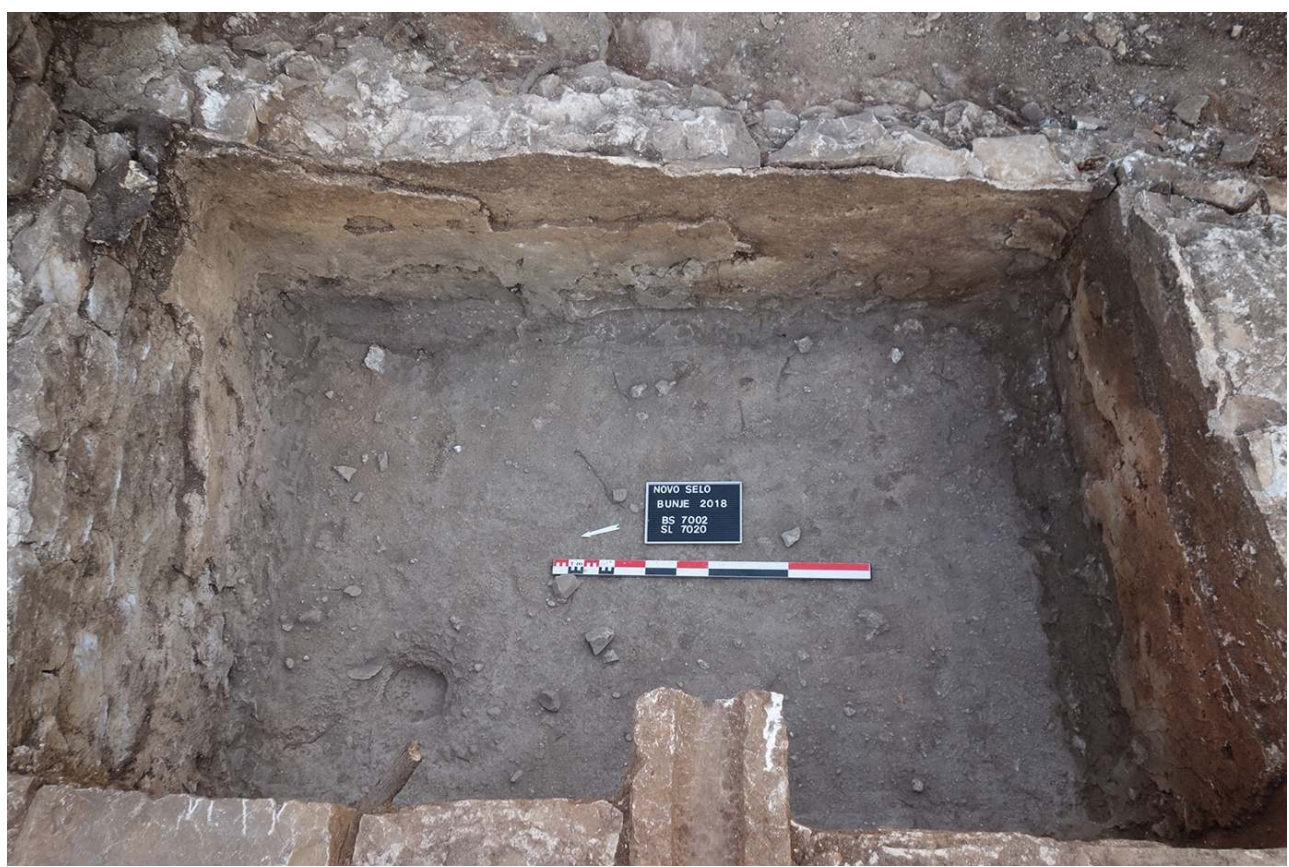

Cl. E. Botte.

9 Le bassin occupe l'angle nord-ouest d'une grande pièce rectangulaire $(12 \times 5,40 \mathrm{~m}$, fig. 7) qu'il est raisonnable de considérer comme le chai de la villa, qui a pu également servir au 
stockage de l'huile d'olive dans une autre phase. La position de cet espace dans la partie productive de la villa est en adéquation avec cette fonction, comme l'attestent de nombreux exemples en Méditerranée antique. Nous avons réalisé une série de sondages pour déterminer si le vin y était stocké dans des dolia, qui en général, même s'ils ont été récupérés, laissent l'empreinte de leur présence dans le sol. Or aucune trace n'en a été décelée, et nous supposons alors que la vinification ne s'effectuait pas dans des dolia mais dans des tonneaux, comme c'est le cas notamment dans plusieurs villae du nord de la Gaule $^{3}$. La datation de la villa de Bunje est en adéquation avec l'utilisation de ce mode de vinification, qui ne semble pas antérieur au $\mathrm{I}^{\mathrm{er}}$ siècle de notre ère.

Fig. 7 - Vue depuis le sud de l'espace du chai de la villa.

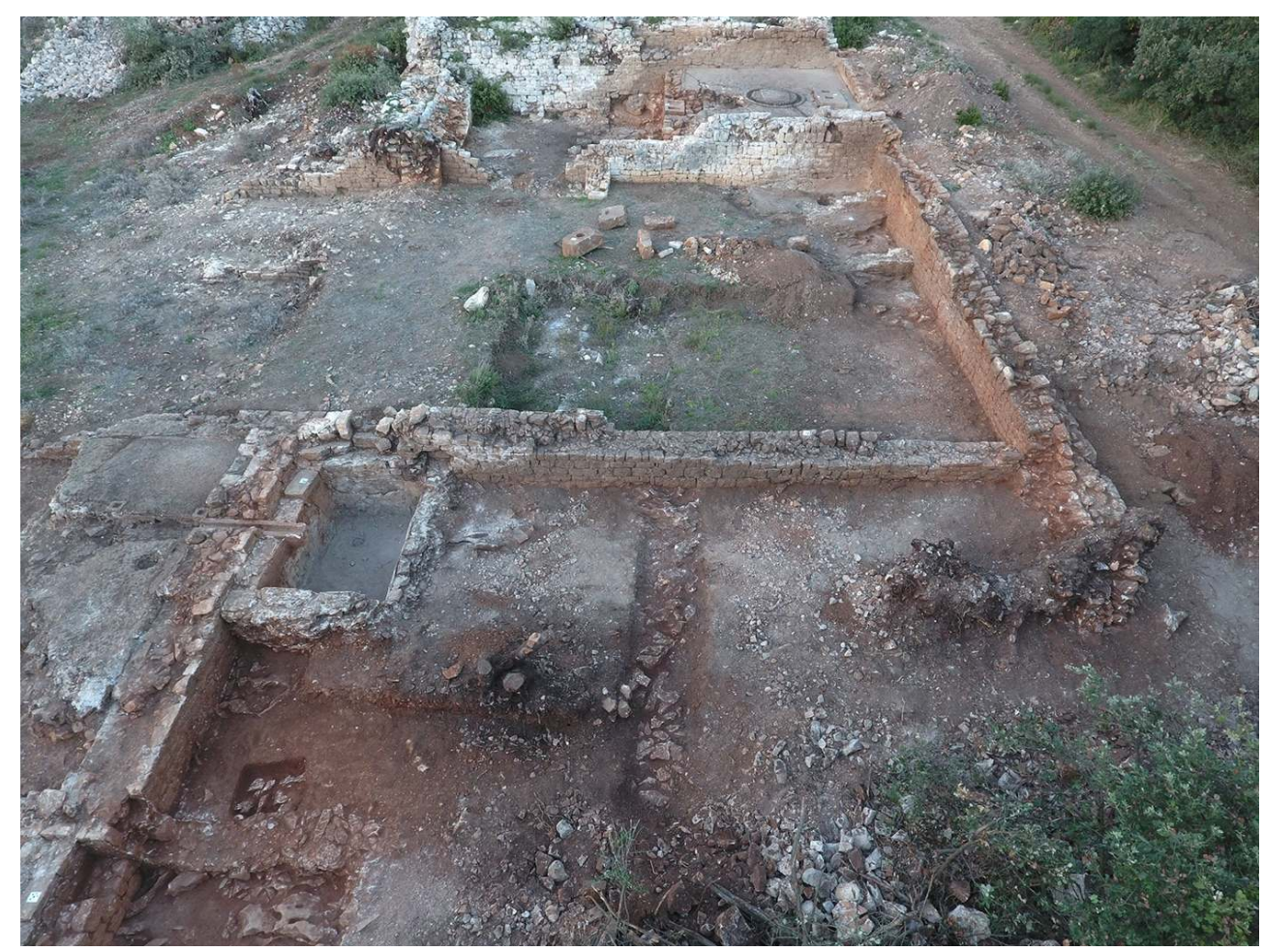

Cl. E. Botte.

Nous avons également procédé au dégagement de la zone directement au sud de cet espace. Plusieurs murs sont apparus, dans un axe nord-sud, dont un qui s'appuie sur le grand mur méridional de la villa (MR 7023, fig. 8). Il faut en conclure que, contrairement à ce que nous pensions lors des premières campagnes de fouille, les murs s'appuyant sur la face méridionale de ce grand mur ne sont pas des contreforts mais s'avèrent appartenir à une terrasse inférieure. Nous pouvons désormais en être sûrs car un des deux murs découverts cette année correspond au mur de l'espace dégagé au sud de la villa lors de la précédente campagne. Ainsi la campagne de l'année 2019 aura pour objectif de faire la liaison entre ces deux terrasses pour en déterminer, en fonction de l'état de conservation, les procédés de circulation entre elles et essayer de comprendre si la terrasse inférieure correspond au plan d'origine de la grande villa construite dans le second tiers du II ${ }^{\mathrm{e}}$ siècle de notre ère, ou si elle a été ajoutée dans une phase postérieure. Ces dégagements devraient également nous permettre de comprendre comment s'opère l'installation du sarcophage dans ce secteur au cours du $\mathrm{V}^{\mathrm{e}}$ siècle de notre ère, et de percevoir sa place par rapport au reste de la villa encore occupée durant cette période. 
Fig. 8 - Vue depuis l'ouest du mur MR 7023 appartenant à la terrasse inférieure.

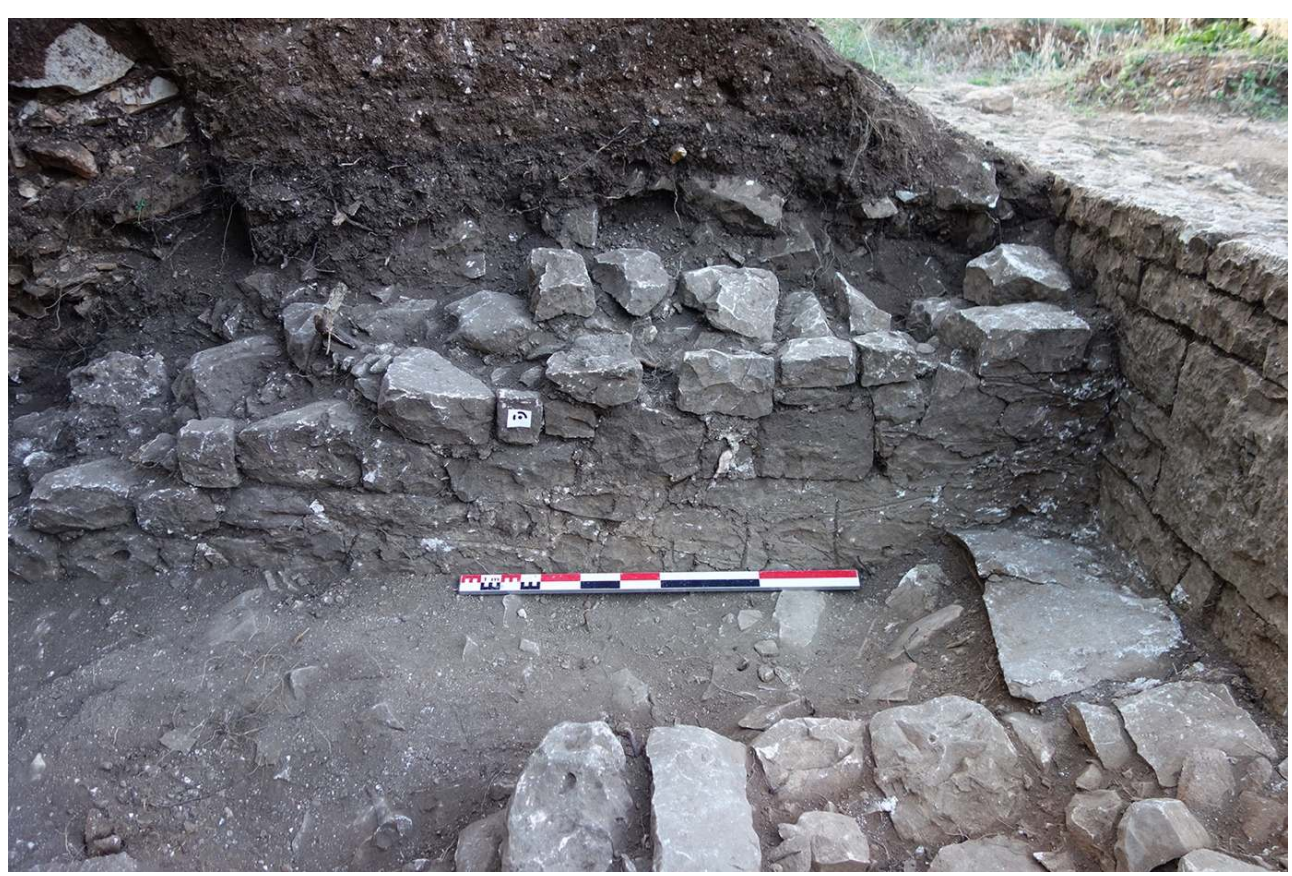

Cl. E. Botte

\section{Secteur 8}

Dans le secteur 8, qui correspond à celui du pressoir et de l'espace directement au sud, nous avons poursuivi les dégagements afin de mettre au jour le mur oriental de la villa. L'espace au sud du pressoir, dans lequel nous avions découvert le bassin de récolte du pressoir dans l'angle nord-est, s'est révélé pauvre d'un point de vue stratigraphique. Les dégagements ont uniquement permis de mettre au jour différents murs appuyés contre la face ouest du mur oriental de la villa, sans beaucoup nous éclairer sur la nature des espaces qu'ils délimitent. L'espace n'étant pas encore entièrement dégagé, nous pouvons espérer obtenir une partie des réponses durant la campagne de cette année.

Dans le secteur du pressoir, les nettoyages et le sondage effectués au contact de la maie nous ont apporté plusieurs informations importantes. Nous avons ainsi effectué un sondage dans la partie la plus abîmée du pressoir, au contact de la partie occidentale de la maie. Cela nous a permis d'observer que sous le niveau de béton de tuileau se trouvait le hérisson qui le supportait, et que ce dernier était posé directement sur le sol, également en béton de tuileau, d'un pressoir antérieur (fig. 9). La limite occidentale de ce pressoir a également pu être observée car nous avons découvert son mur de limite, MR 8098 (fig. 10 ). Ce pressoir mesurait donc 5,20 × 3,85 m, et était déjà doté d'un canal traversant le mur sud MR 8073 pour se jeter dans le bassin BS 8045. Dans une seconde phase, le bloc des jumelles du premier état est récupéré et intégré dans la construction du mur de limite occidentale du pressoir postérieur (MR 8074). Ce pressoir, plus grand $(5,20 \times 5 \mathrm{~m})$ est équipé d'un bloc de jumelles plus grand et appartient à la typologie des pressoirs à treuil et câbles, qui est le modèle le plus attesté en Istrie et Dalmatie à ce jour (voir notamment celui de Kapljuk sur le site de Salone où un pressoir à huile de plan identique a été mis au jour [fig. 11]). Nous avons procédé au dégagement de la face ouest du mur MR 8074, qui 
nous a permis de découvrir à son pied trois monnaies dont une dans un excellent état de conservation (fig. 12). Il s'agit d'une monnaie de Constance II, qui règne entre 337 et 361 . Si cette monnaie est contemporaine du remaniement du pressoir, cela indiquerait que celui-ci est réalisé durant le second tiers du IV ${ }^{\mathrm{e}}$ siècle de notre ère. Nous n'avons en revanche pas d'éléments concernant la datation du premier pressoir, mais dans l'état actuel de la documentation, nous sommes tentés de le faire appartenir à la villa aménagée dans le second tiers du $\mathrm{II}^{\mathrm{e}}$ siècle de notre ère.

Fig. 9 - Vue du sol en béton de tuileau du premier pressoir.

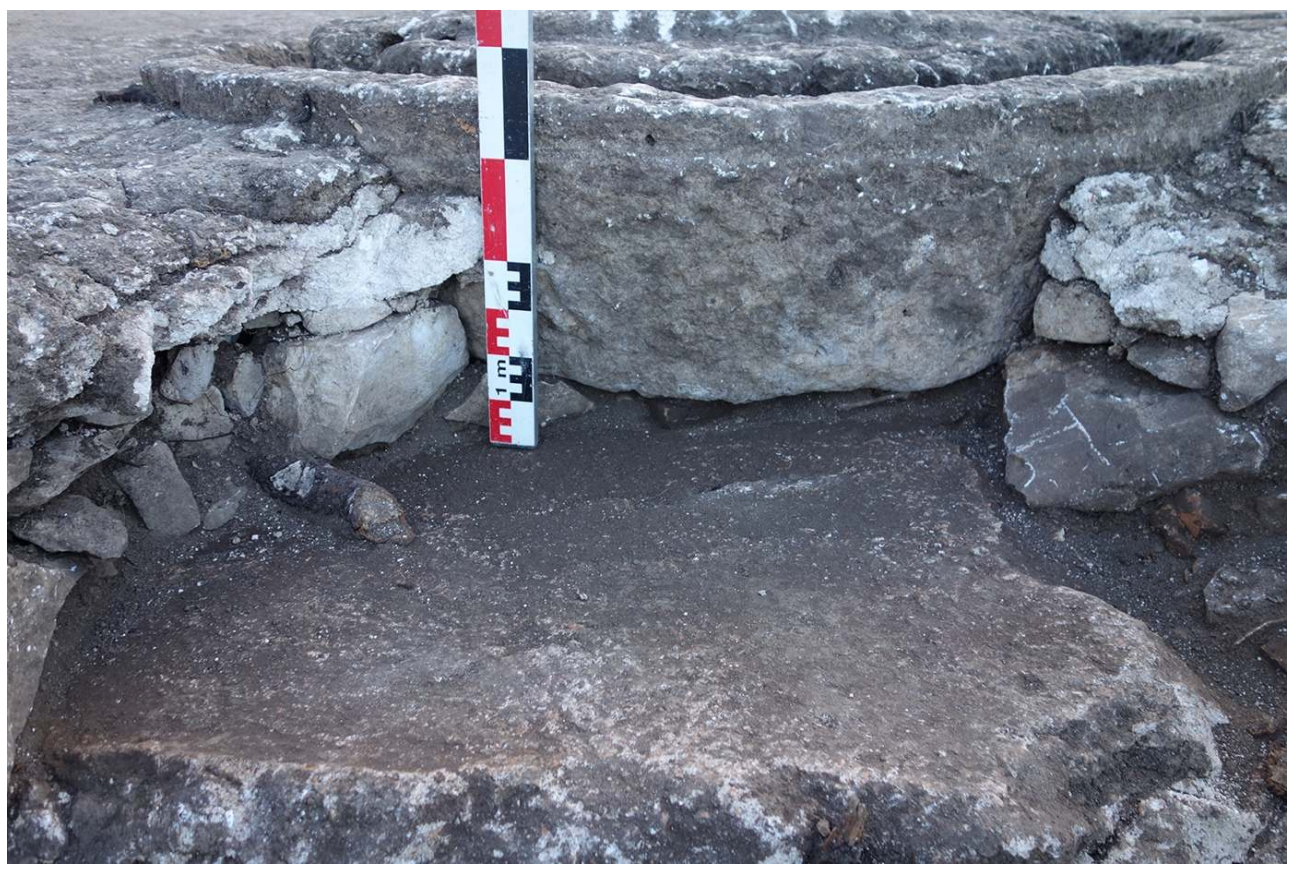

Cl. E. Botte. 
Fig. 10 - Vue par drone de la superposition des deux pressoirs.

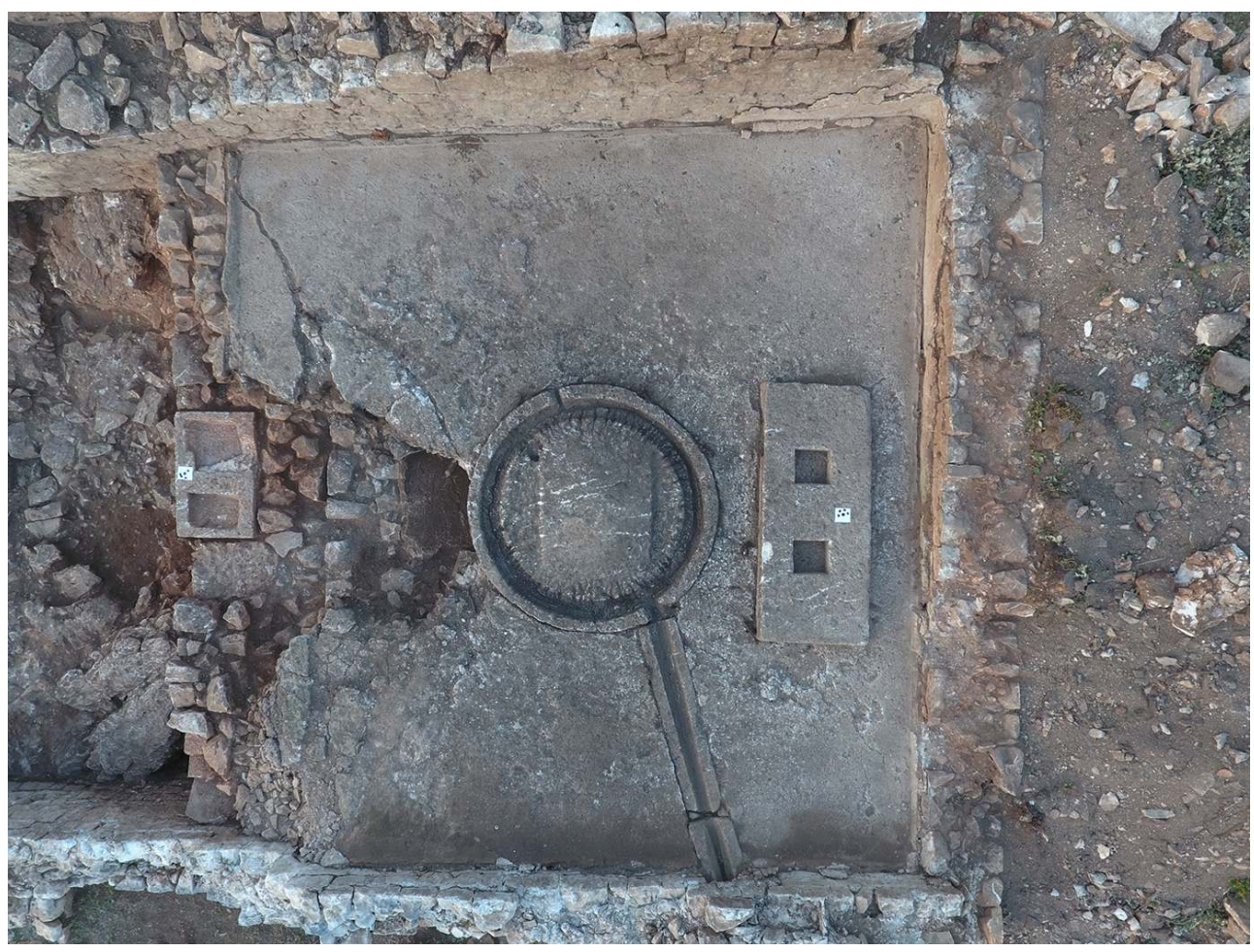

Cl. E. Botte

Fig. 11 - Vue du pressoir de l'Antiquité tardive de Kapljuk à Salone.

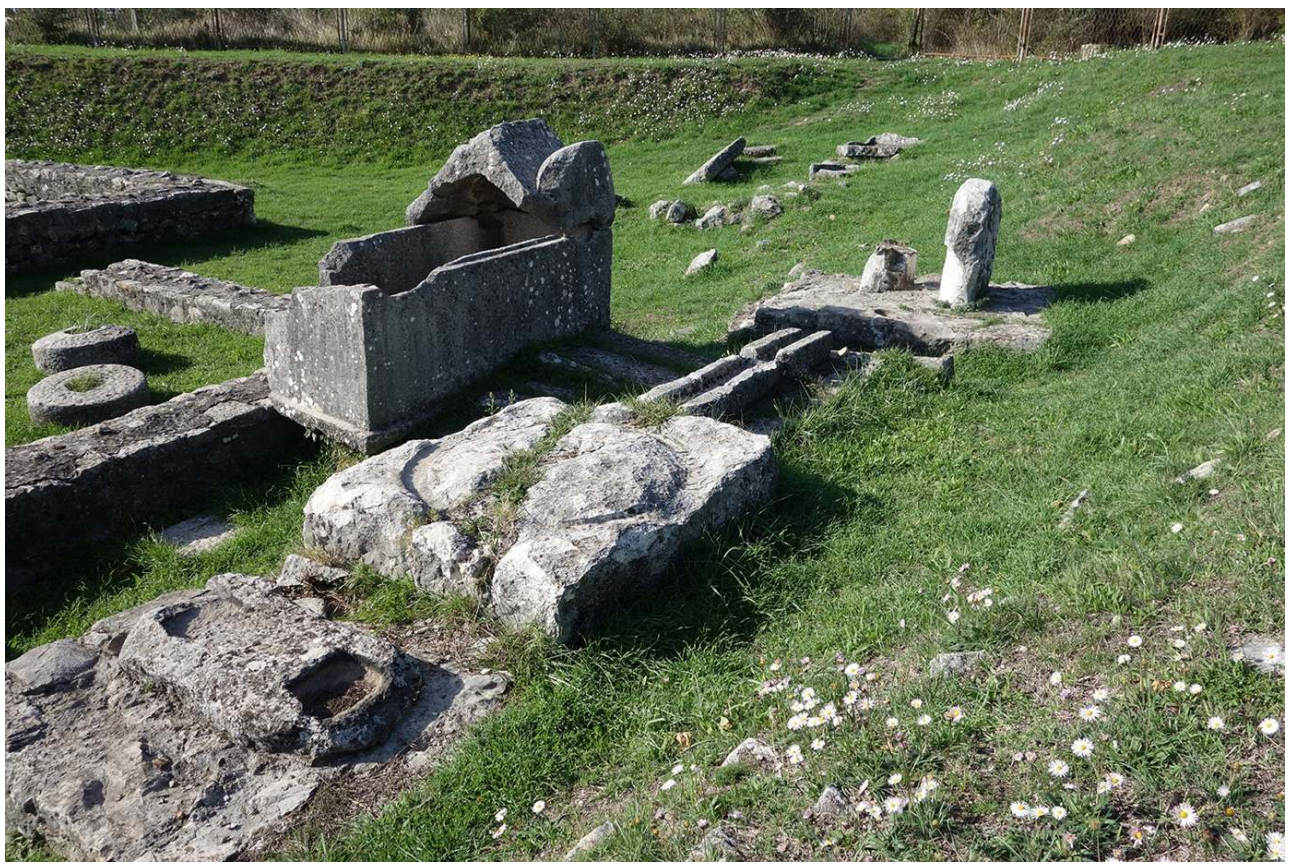

Cl. E. Botte. 
Fig. 12 - Monnaie de Constance II découverte au pied du pressoir de l'Antiquité tardive.

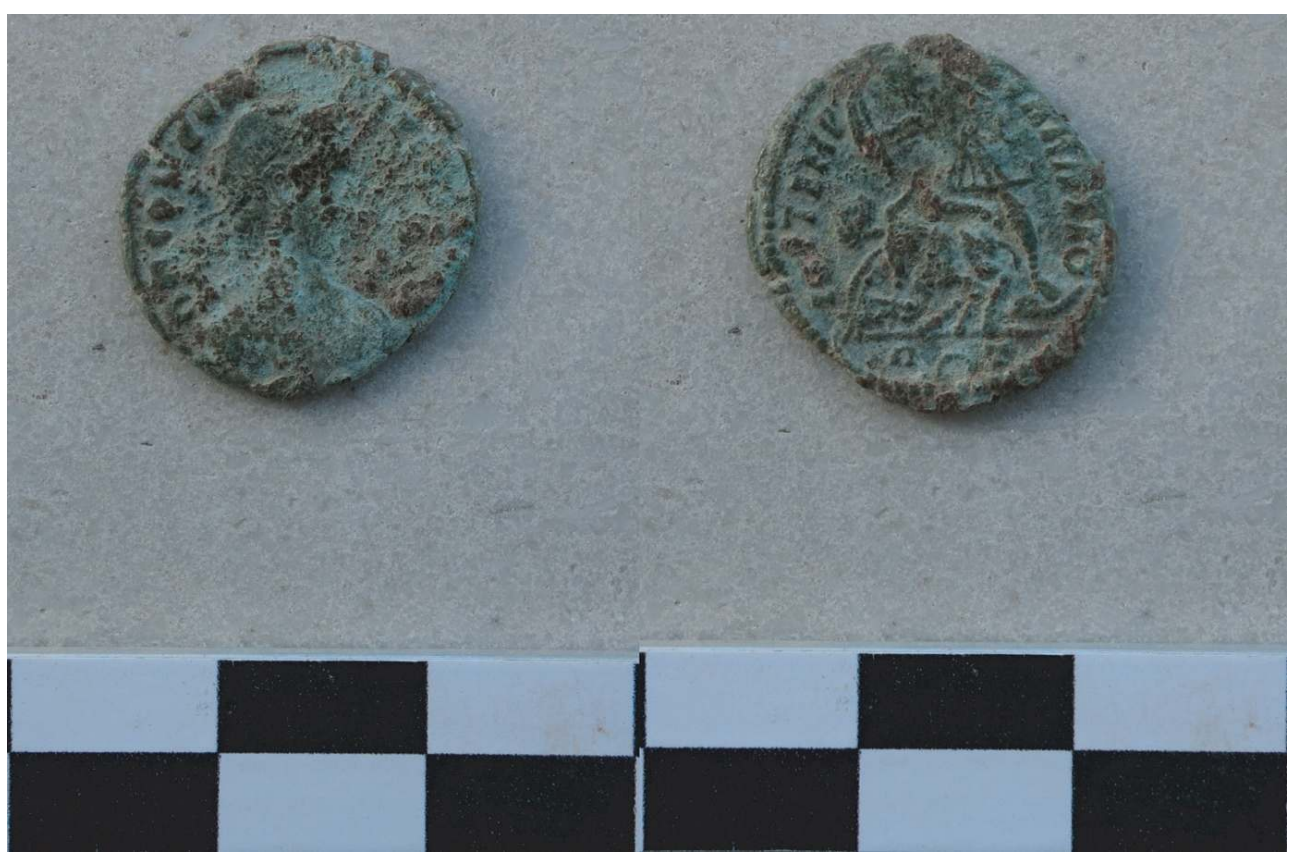

Cl. E. Botte.

\section{Synthèse et programme des prochaines campagnes}

13 Cette brève campagne de fouilles nous a permis de faire des avancées importantes dans la compréhension de l'organisation de la villa (fig. 13), puisque nous avons pu constater qu'elle était organisée en un système de terrasses (fig. 14-15). Il nous reste désormais à comprendre comment s'effectue la circulation entre ces deux terrasses, et si la terrasse inférieure appartient au plan général de la grande villa dès sa construction au $\mathrm{II}^{\mathrm{e}}$ siècle de notre ère ou s'il s'agit d'un ajout dans une phase postérieure. Nos connaissances ont par ailleurs également progressé du côté de la pars rustica de la villa. Nous avons pu constater qu'elle est équipée d'un fouloir et d'un pressoir dès sa construction. Dans une seconde phase, les dimensions de ces deux structures sont agrandies, indiquant alors une augmentation de la production en termes de volume. 
Fig. 13 - Plan général de la terrasse principale de la villa à la fin de la campagne de fouilles.

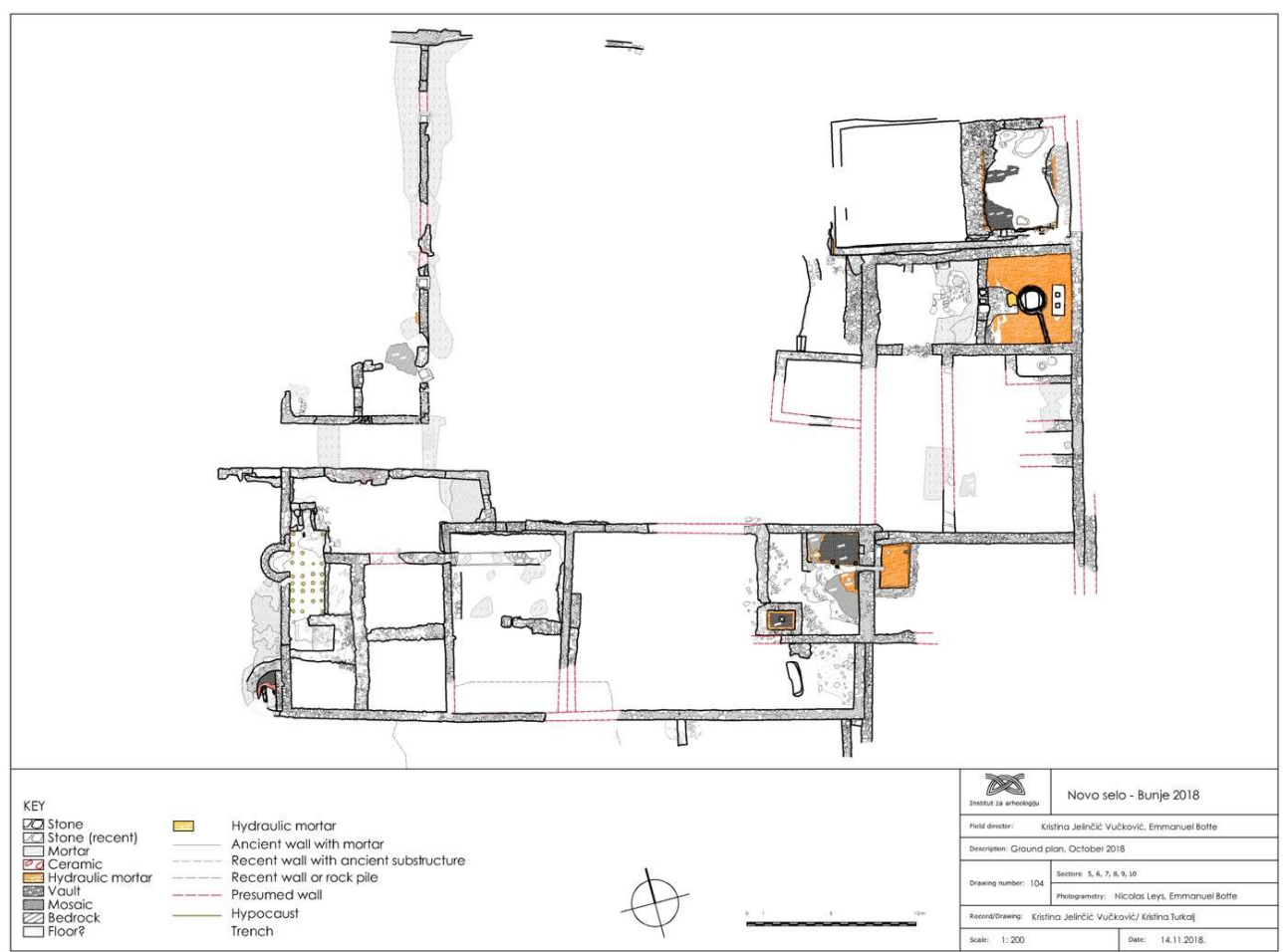

Plan K. Jelinčić.

Fig. 14 - Plan de l'ensemble de la villa avec les deux terrasses à la fin de la campagne de fouilles.

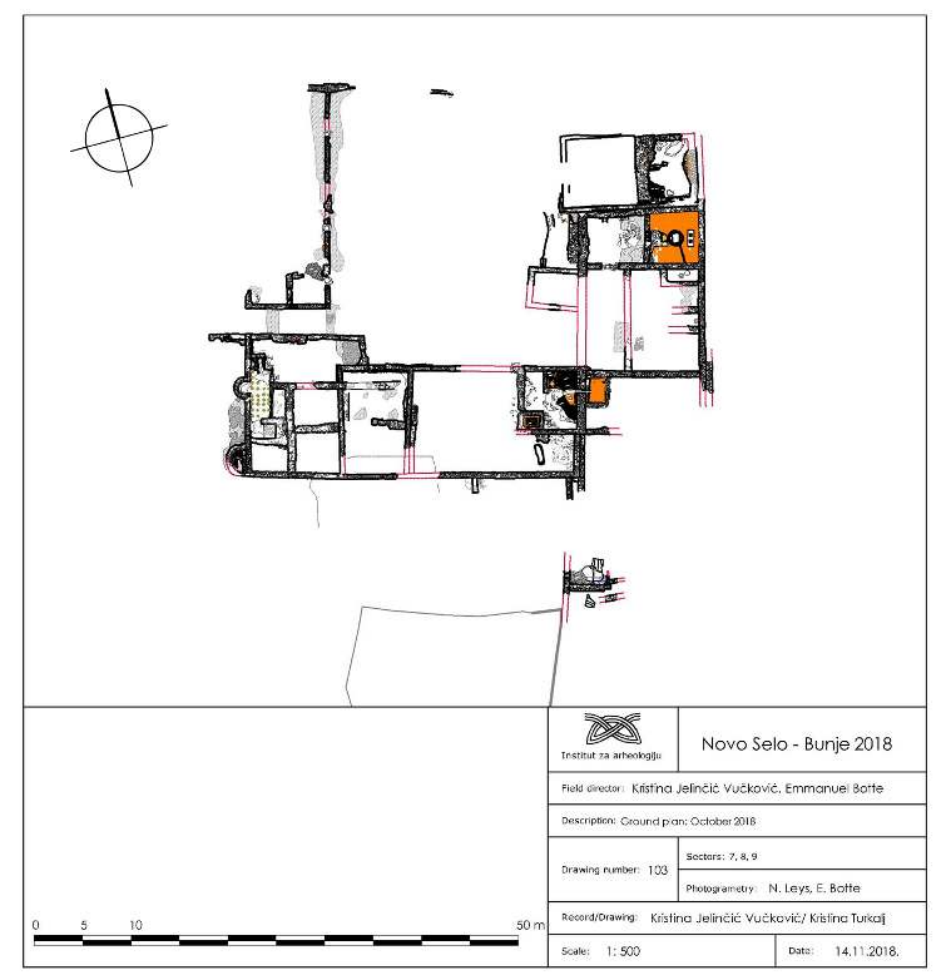

Plan K. Jelinčić. 
Fig. 15 - Vue par drone depuis le Sud de la villa de Bunje à la fin de la campagne de fouilles.

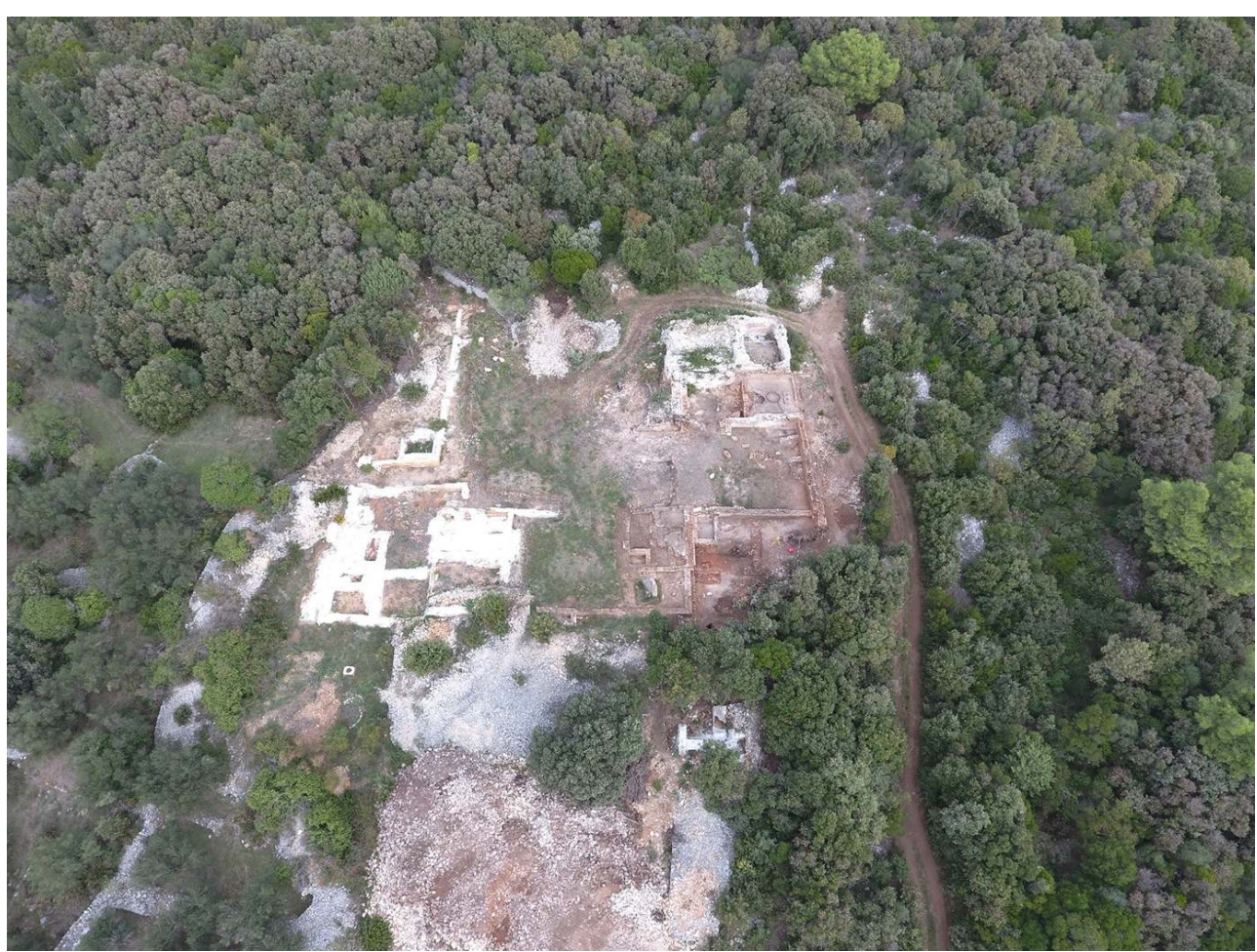

Cl. E. Botte.

La prochaine campagne de fouilles sera destinée à dégager la terrasse inférieure de la villa afin d'analyser sa relation avec la terrasse supérieure et principale de l'édifice. Nous espérons également que nous pourrons déterminer la fonction des espaces qui s'y trouvent.

\section{BIBLIOGRAPHIE}

Botte - Bertrand - Jelinčić 2016 = E. Botte, A. Bertrand, K. Jelinčić, Bunje (Novo Selo, Croatie), dans Chronique des activités archéologiques de l'École française de Rome [en ligne]. DOI : 10.4000/cefr.1519

Botte - Jelinčić 2017 = E. Botte, K. Jelinčić, Bunje (Novo Selo, Croatie), dans Chronique des activités archéologiques de l'École française de Rome [en ligne]. DOI : 10.4000/cefr.1723

Botte et al. 2019 = E. Botte, A. Bertrand, K. Jelinčić, N. Leys, A. Boisson, Bunje (Novo Selo, Croatie), campagnes 2017-2018, dans Chronique des activités archéologiques de l'École française de Rome [en ligne]. DOI : $10.4000 /$ cefr. 2419

Brun 2005 = J.-P. Brun, Archéologie du vin et de l'huile en Gaule romaine, Paris, 2005.

Jelinčić $2012=\mathrm{K}$. Jelinčić, Izvješće arheološkom rekognosciranju i snimanju lokaliteta Novo Selo Bunje (18.-19. travnja 2012.), Institut za arheologiju, Zagreb, 2012. 


\section{NOTES}

1. Pour un résumé des résultats des campagnes précédentes, voir Botte Bertrand - Jelinčić 2017 ; Botte - Jelinčić 2018 ; Botte et al. 2019.

2. Jelinčić 2012.

3. En fait dès que l'on se trouve hors de la Gaule Narbonnaise, voir des exemples dans Brun 2005 , p. 106-117.

INDEX

institutions École française de Rome, Ministère de l'Europe et des Affaires étrangères, Institut Archéologique de Zagreb, Centre Camille Jullian, Université Paris-Est Marne, mairie de Selca Index géographique : Bunje

Mots-clés : Dalmatie, villa romaine, vin, huile, Antiquité

\section{AUTEURS}

\section{EMMANUEL BOTTE}

Aix Marseille Univ, CNRS, CCJ, Aix-en-Provence, France

\section{AUDREY BERTRAND}

Université Paris Est Marne-la-Vallée

\section{KRISTINA JELINČIĆ}

Institut archéologique de Zagreb

\section{BASTIEN LEMAIRE}

Université Paul Valéry Montpellier 3

NICOLAS LEYS

Sorbonne Université - Institut des Sciences du Calcul et des Données

\section{ANTOINE BOISSON}

Université Paul Valéry Montpellier 3 